\title{
Effect of sulfate on methanogenic communities that degrade unsaturated and saturated long-chain fatty acids (LCFA)
}

Diana Z. Sousa, ${ }^{1,2 \star}$ Joana I. Alves, ${ }^{1,2}$ M. Madalena Alves, ${ }^{1}$ Hauke Smidt ${ }^{2}$ and Alfons J. M. Stams ${ }^{2}$

${ }^{1}$ IBB - Institute for Biotechnology and Bioengineering, Centre of Biological Engineering, University of Minho, 4710-057 Braga, Portugal.

${ }^{2}$ Laboratory of Microbiology, Wageningen University, Dreijenplein 10, 6703 HB Wageningen, the Netherlands.

\section{Summary}

Anaerobic bacteria involved in the degradation of long-chain fatty acids (LCFA), in the presence of sulfate as electron acceptor, were studied by combined cultivation-dependent and molecular techniques. The bacterial diversity in four mesophilic sulfate-reducing enrichment cultures, growing on oleate $\left(\mathbf{C}_{18: 1}\right.$, unsaturated LCFA) or palmitate $\left(\mathbf{C}_{16: 0}\right.$, saturated LCFA), was studied by denaturing gradient gel electrophoresis (DGGE) profiling of polymerase chain reaction (PCR)-amplified 16S rRNA gene fragments. These enrichment cultures were started using methanogenic inocula in order to assess the competition between methanogenic communities and sulfate-reducing bacteria. Phylogenetic affiliation of rRNA gene sequences corresponding to predominant DGGE bands demonstrated that members of the Syntrophomonadaceae, together with sulfate reducers mainly belonging to the Desulfovibrionales and Syntrophobacteraceae groups, were present in the sulfate-reducing enrichment cultures. Subculturing of LCFA-degrading methanogenic cultures in the presence of sulfate resulted in the inhibition of methanogenesis and, after several transfers, archaea could no longer be detected by real-time PCR. Competition for hydrogen and acetate was therefore won by sulfate reducers, but acetogenic syntrophic bacteria were the only known LCFA-degrading organisms present after subculturing with sulfate. Principal component analysis of the DGGE profiles from methanogenic and

Received 14 January 2008; accepted 20 July, 2008. *For correspondence. E-mail dianasousa@ deb.uminho.pt; Tel. (+351) 253 604 400; Fax (+351) 253678986 sulfate-reducing oleate- and palmitate-enrichment cultures showed a greater influence of the substrate than the presence or absence of sulfate, indicating that the bacterial communities degrading LCFA in the absence/presence of sulfate are rather stable.

\section{Introduction}

Long-chain fatty acids (LCFA) are the main products of lipids hydrolysis, and are commonly found in several types of wastewaters. Through effective anaerobic treatment, LCFA can be microbiologically converted to large amounts of biogas (Pereira et al., 2002a; 2003; 2004), a renewable source of energy. However, in wastewaters containing sulfate, the potential for the energetic valorization of LCFA through production of methane-rich biogas can decrease due to a total or partial inhibition of methanogenesis. Sulfate is a widespread compound in some of the LCFA-rich wastewaters, as the ones produced in slaughterhouses, food-processing industries and edible oil production units. In methanogenic environments, LCFA are completely degraded to methane and carbon dioxide by complex syntrophic communities involving acetogenic syntrophic bacteria and methanogenic acetate- and hydrogen-consuming archaea (Sousa et al., 2007a). However, in wastewaters containing sulfate, sulfate-reducing bacteria (SRB) can out-compete syntrophic acetogens for fatty acids and methanogens for hydrogen and/or acetate (Colleran et al., 1995).

Many SRB can incompletely oxidize LCFA to acetate or acetate plus propionate or, ultimately, to $\mathrm{CO}_{2}$ and sulfide (Rabus et al., 2006), with no need of a syntrophic partner. The ability to degrade LCFA is widespread among SRB, and has been shown for members of 15 different genera within the Desulfobacterales, Desulfuromonadales, Syntrophobacterales and Clostridiales orders (Rabus et al., 2006; Rees and Patel, 2001). These bacteria were isolated from marine and freshwater sediments and from oil field waters. Their presence in anaerobic reactors treating lipids- and LCFA-based wastewaters, however, was never reported. Conversely, hydrogen- and acetate-consuming SRB are commonly found in anaerobic sludge reactors. Competition for hydrogen between methanogens and SRB has been reported to occur in anaerobic reactors 
treating different effluents (Isa et al., 1986; Visser et al., 1993; Harada et al., 1994; O'Flaherty et al., 1998). Reports on the competition of methanogenic aceticlastic archaea and SRB are more contradictory; a predominance both of SRB (Alphenaar et al., 1993; Stucki et al., 1993; Gupta et al., 1994; Oude Elferink et al., 1995) and of methanogens (Isa et al., 1986; Visser et al., 1993; Omil et al., 1996; O'Flaherty et al., 1998) has been observed.

To date, little is known about the microbial communities involved in the degradation of LCFA in anaerobic bioreactors with high levels of sulfate. Also, there are no reports on the potential competition of syntrophs and methanogens with SRB during the degradation of LCFA in this type of systems. In this work, a combined approach of molecular microbial ecology techniques and cultivation experiments was used to study the effect of sulfate on the microbial composition of LCFA-degrading methanogenic communities. Bacterial community dynamics in distinct enrichment series, growing on oleate (unsaturated, $\mathrm{C}_{18: 1}$ ) or palmitate (saturated, $\mathrm{C}_{16: 0}$ ), were studied by $16 \mathrm{~S}$ rRNA gene denaturing gradient gel electrophoresis (DGGE) fingerprinting analysis and subsequent sequence analysis. Competition between SRB and methanogens in these enrichment cultures was surveyed by relative quantification of archaea, using real-time polymerase chain reaction (PCR). This provided insight into microbial communities involved in LCFA degradation in the presence of sulfate, including the competition between methanogens and sulfate reducers during this process.

\section{Results}

\section{Molecular profiling of the bacterial communities}

Four distinct sulfate-reducing oleate- and palmitateenrichment cultures were obtained through repeated transfers into fresh medium containing $1 \mathrm{mM}$ oleate $\left(\mathrm{C}_{18: 1}\right.$, unsaturated LCFA) or $1 \mathrm{mM}$ palmitate $\left(\mathrm{C}_{16: 0}\right.$, saturated LCFA) plus $10 \mathrm{mM}$ sodium sulfate. Enrichment series OS and PS (O-oleate; P-palmitate; S-sulfate) were inoculated with anaerobic sludge, sludge I, while specialized and well-characterized oleate and palmitate enrichments, cultures OM-12 and PM-12 (12 corresponds to the number of transfers of the methanogenic enrichments previous to their use as inoculum in this work), were used to start up enrichment series $\mathrm{OM} \rightarrow \mathrm{OS}$ and $\mathrm{PM} \rightarrow \mathrm{PS}$ (see Fig. $\mathrm{S} 1$ for a schematic representation of the experiment set-up). Substrate degradation by the different enrichment cultures (series OS, PS, OM $\rightarrow \mathrm{OS}$ and $\mathrm{PM} \rightarrow \mathrm{PS}$ ) was observed by the clearing of the liquid. Addition of oleate and palmitate to the medium causes an immediate turbidwhite appearance, which disappears when the substrate is consumed. After 3-4 weeks of incubation of the enrichment cultures, turbidity of the medium decreased and sulfide could be detected (data not shown), indicating oleate/palmitate degradation. This shift in appearance and the detection of sulfide were the decision parameters to transfer the cultures to fresh medium. In all the four enrichment series, sulfate reduction started during the first incubation with sulfate (OS-1, PS-1, OM $\rightarrow$ OS-1 and $P M \rightarrow P S-1)$, and after four successive transfers in the presence of sulfate (OS-4, PS-4, OM $\rightarrow$ OS-4 and PM $\rightarrow$ PS-4), no methane could be detected in the bottles' headspace (data not shown). Stable sulfate-reducing enrichment cultures (after four or more successive transfers) were quantitatively analysed for sulfide and volatile fatty acids (VFA) concentrations. Average sulfide concentrations in the medium (after approximately 4 weeks of incubation) were $5.2 \pm 1.1$ and $7.7 \pm 2.4 \mathrm{mM}$ for the oleate- and palmitate-enrichment cultures respectively. Acetate, up to $2 \mathrm{mM}$, was the only VFA accumulating in the sulfate-reducing oleate-enrichment cultures; no VFA were detected in the palmitate enrichments. Analysis of the bacterial communities developed in the OS and PS enrichment cultures, as well as changes in the bacterial community composition of methanogenic oleate- and palmitate-enrichment cultures incubated with sulfate, was performed by using PCR amplification of bacterial $16 S$ rRNA gene fragments followed by DGGE fingerprinting.

Sulfate-reducing OS (Fig. 1A) and PS (Fig. 1C) cultures showed gradually less diverse DGGE patterns over successive transfers in the presence of oleate or palmitate, which is consistent with an average decrease in the bacterial diversity upon enrichment. Oleate-enrichment samples taken after four subsequent transfers (OS-4 to OS-7) showed an average Shannon diversity index $(H)$ of $1.63 \pm 0.08$, which represents a decrease of $40 \pm 3 \%$ compared with the initial bacterial diversity in the inoculum ( $H$ of 2.73). Similarly, average bacterial diversity in samples PS-4 to PS-7 $(2.30 \pm 0.16)$ decreased by $16 \pm 6 \%$ in comparison with the inoculum sludge. An evident shift in the bacterial community structure was already observed after the first transfer in the presence of oleate or palmitate (Fig. 1A, lane OS-1 and Fig. 1C, lane PS-1 respectively), when compared with the inoculum (Fig. $1 \mathrm{~A}$ and $\mathrm{C}$, lanes sludge I). Pearson similarity coefficients of $12 \%$ and $34 \%$ between the inoculum and samples OS-1 and PS-1, respectively, were obtained. DGGE profiles of the oleate-enrichment culture from the fourth transfer onwards (OS-4 to OS-7) appeared to be rather stable with an average Pearson similarity of $84 \pm 2 \%$. A relatively lower average Pearson similarity was found between palmitate-enrichment samples PS-4 to PS-7, i.e. $54 \pm 16 \%$. Although the same inoculum was used for starting up OS and PS enrichment series, DGGE profiles obtained after enrichment with either substrate were clearly different (Fig. 1), and Pearson similarity between samples OS-7 and PS-7 was as low as $16 \%$. 


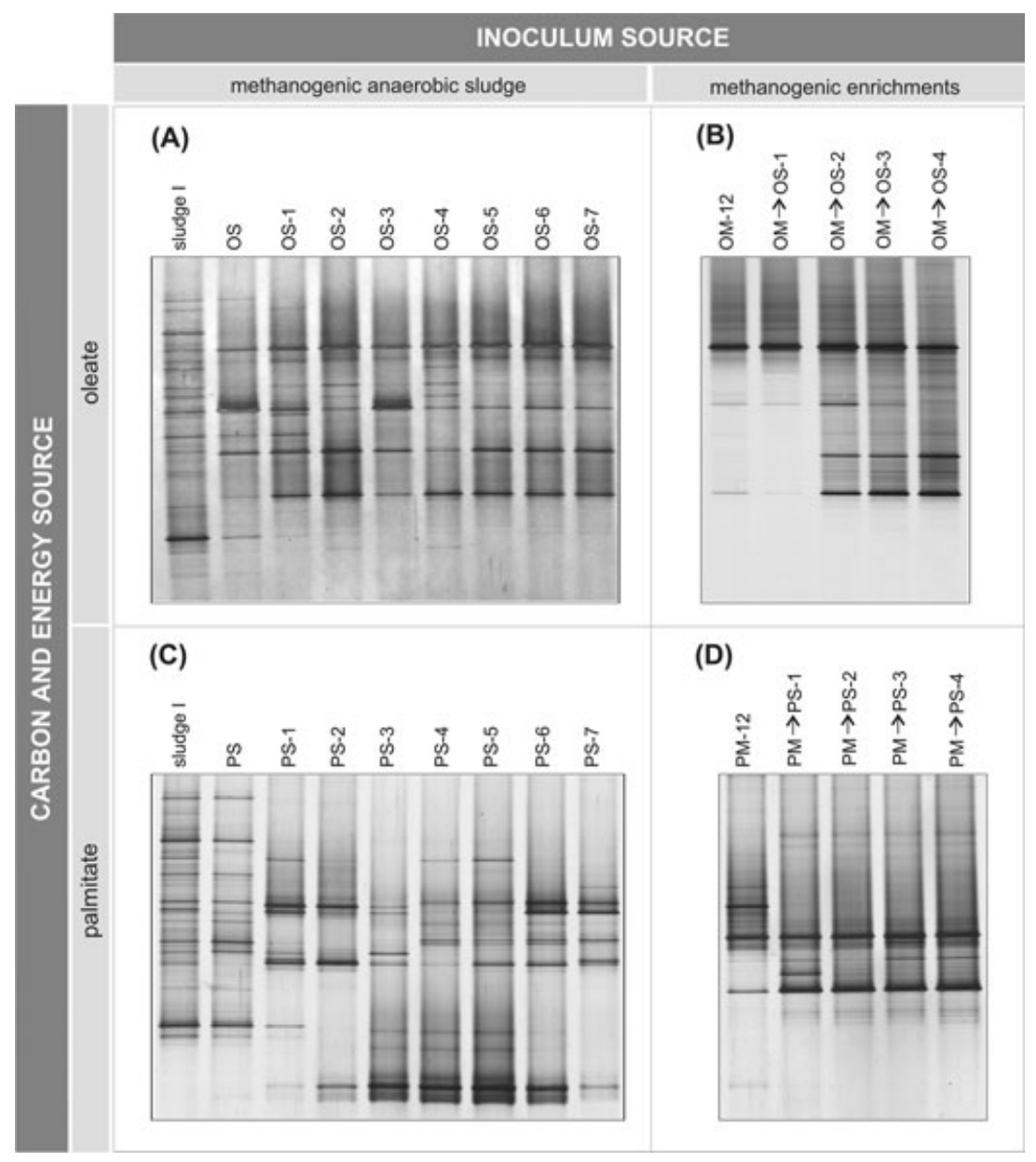

Fig. 1. Bacterial DGGE patterns showing the evolution of the four distinct sulfate-reducing enrichments over time: (A) OS enrichment series, (B) $\mathrm{OM} \rightarrow \mathrm{OS}$ enrichment series, (C) $P S$ enrichment series and (D) $P M \rightarrow P S$ enrichment series. These cultures were obtained by successive transfers in the presence of $1 \mathrm{mM}$ oleate or palmitate and $10 \mathrm{mM}$ sulfate (sludge I, OM-12 and PM-12 inocula; OS- $x$ and OM $\rightarrow$ OS- $x-$ oleate-enrichment cultures; PS- $x$ and $\mathrm{PM} \rightarrow \mathrm{PS}-x-$ palmitate-enrichment cultures; $x$ represents the number of transfers).

Methanogenic enrichment cultures used as inoculum for $\mathrm{OM} \rightarrow \mathrm{OS}$ and $\mathrm{PM} \rightarrow \mathrm{PS}$ enrichment series showed already a low bacterial diversity $-H$ of 1.21 and 1.96 for cultures OM-12 and PM-12 respectively (Fig. 1B and D). In the presence of sulfate, the bacterial diversity in the oleate-enrichment culture slightly increased $(H$ of 1.63 for sample $\mathrm{OM} \rightarrow \mathrm{OS}-4)$. Conversely, in the palmitateenrichment series the bacterial diversity index for culture $\mathrm{PM} \rightarrow \mathrm{PS}-4$ was lower, i.e. $H 1.22$, than in the corresponding inoculum. For both sulfate-reducing enrichment series, $\mathrm{OM} \rightarrow \mathrm{OS}$ and $\mathrm{PM} \rightarrow \mathrm{PS}$, a shift in the bacterial communities was observed (Pearson similarity coefficients of $53 \%$ and $62 \%$ between $\mathrm{OM}-12 / \mathrm{OM} \rightarrow \mathrm{OS}-4$ and $\mathrm{PM}-12 / \mathrm{PM} \rightarrow \mathrm{PS}-4$ respectively), suggesting an adaptation of the communities to the presence of sulfate.

\section{Structure of the bacterial communities}

Predominant bands from stable sulfate-reducing oleate and palmitate enrichments were identified by cloning and sequencing of the 16S rRNA gene (Fig. 2, OS-7 and PS-7). A total of 168 and 125 transformants were obtained from OS-7 and PS-7 enrichments respectively. V6 to V8 regions of the cloned 16S rRNA gene of cell lysates with different ARDRA profiles were further amplified, and electrophoretic mobility of these amplicons was compared with DGGE profiles of the oleate- and palmitateenrichment samples. Amplicons with the same mobility as predominant DGGE bands in the oleate- and palmitateenrichment profiles were selected for sequence analysis. Supporting the semi-quantitative value of DGGE analysis, predominant bands in DGGE profiles were found to correspond to most abundant ARDRA ribotypes observed in the clone libraries. A total of $72 \%$ and $66 \%$ of the clones retrieved from the enrichment cultures could be assigned to the predominant bands in the DGGE profiles of the oleate- and palmitate-enrichment cultures respectively. Phylogenetic affiliation of the retrieved sequences was performed by secondary structure-assisted alignment (Fig. 3).

Phylogenetic analysis showed that both sulfatereducing oleate- and palmitate-enrichment cultures were mainly composed of syntrophic acetogenic bacteria belonging to the Syntrophomonadaceae, together with sulfate reducers belonging to the Desulfovibrionales and Syntrophobactereacea clusters (Fig. 3). Similarly, a previ- 


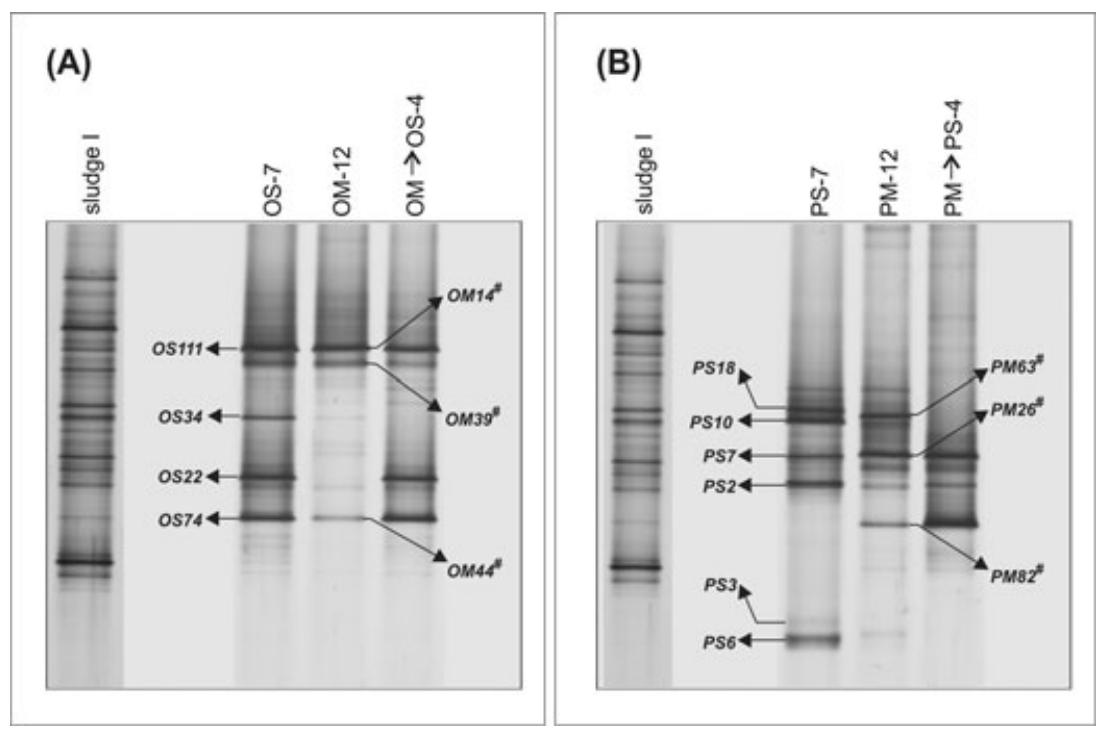

Fig. 2. Bacterial DGGE profiles of (A) oleateand (B) palmitate-enrichment cultures. A. Stable sulfate-reducing (OS-7) and methanogenic $(\mathrm{OM}-12)$ oleate-enrichment cultures deriving directly from sludge I. Sulfate-reducing enrichment culture $\mathrm{OM} \rightarrow \mathrm{OS}-4$ was obtained from culture $\mathrm{OM}-12$ after four successive transfers in the presence of sulfate.

B. Stable sulfate-reducing (PS-7) and methanogenic (PM-12) palmitate-enrichment cultures deriving directly from sludge $\mathrm{I}$. Sulfate-reducing enrichment culture $\mathrm{PM} \rightarrow \mathrm{PS}-4$ was obtained from culture PM-12 after four successive transfers in the presence of sulfate.

Dominant DGGE bands identified by cloning and sequencing are indicated in the figure; \#Sousa and colleagues (2007b).

ous study on the molecular analysis of methanogenic oleate- and palmitate-enrichment cultures (Sousa et al., 2007b) revealed that syntrophic fatty acid-degrading bacteria belonging to the Syntrophomonadaceae were predominant, and most probably responsible for LCFA degradation in syntrophic interaction with methanogens (see also Fig. 2, lanes OM-12 and PM-12, and Fig. 3).

Clones OS111 and PS18, branching within the syntrophic fatty acid-oxidizing bacteria, share high similarity with sequences obtained from methanogenic oleateand palmitate-enrichment cultures (99\% and $97 \%$ identity between clones OS111/OM14 and PS18/PM63 respectively) (Sousa et al., 2007b; Figs 2 and 3). Clones OM14 and OS111 are closely related to Syntrophomonas zehnderi (99\% 16S rRNA gene identity), a syntrophic oleate-degrading bacterium (Sousa et al., 2007c). DGGE band corresponding to clone OM14 from sample OM-12 persisted as a predominant band even after incubation and successive transfers of the methanogenic oleateenrichment culture in medium containing sulfate (Fig. 2A, lanes $\mathrm{OM}-12$ and $\mathrm{OM} \rightarrow \mathrm{OS}-4)$. Similarly, clone PM26, clustering also within the syntrophic fatty acid oxidizers, maintained its predominance after incubation of the methanogenic palmitate-enrichment culture in the presence of sulfate (Fig. 2, lanes PM-12 and PM $\rightarrow$ PS-4, and Fig. 3). DGGE band corresponding to clone $P M 63$ in the methanogenic palmitate-enrichment culture was virtually absent in the DGGE profile after contact with sulfate (Fig. 2; lanes PM-12 and PM $\rightarrow$ PS-4 respectively). This band represented a probable fatty acid-oxidizing bacteria branching within the Syntrophomonas genus (Sousa et al., 2007b; Fig. 3).

In addition to syntrophic fatty acid-oxidizing bacteria, SRB belonging to the genera Desulfovibrio (clones OS74 and PS2) and Desulfomicrobium (clones OS34 and $P S 10)$ were detected in the sulfate-reducing oleate- and palmitate-enrichment cultures (Figs 2 and 3). The DGGE band corresponding to clone OM44, representing a close relative of Desulfovibrio sp., was already observed in the methanogenic oleate-enrichment culture prior to sulfate addition (Sousa et al., 2007b), but significantly increased in relative intensity after incubation with sulfate (Fig. 2). Additionally, in the oleate-enrichment cultures (OS and $\mathrm{OM} \rightarrow \mathrm{OS}$ ) there was a predominant band corresponding to a Desulforhabdus-like microorganism (clone OS22). This DGGE band was already detected as predominant after the first incubation of the OS cultures with sulfate and appears in the $\mathrm{OM} \rightarrow \mathrm{OS}$ cultures just after two subsequent incubations with sulfate (Figs 1 and 2). The type strain of this genus, Desulforhabdus amnigena, is an acetate-consuming microorganism that can use hydrogen and acetate simultaneously (Oude Elferink et al., 1998). In addition, the related sulfate-reducing microorganism Thermodesulforhabdus norvegica is able to grow on LCFA, such as palmitate $\left(\mathrm{C}_{16: 0}\right)$ and stearate $\left(\mathrm{C}_{18: 0}\right)$, at moderately high temperature (Beeder et al., 1995). Degradation of these LCFA by $D$. amnigena was never tested, although it can grow on the C4-fatty acid butyrate (Oude Elferink et al., 1995). To evaluate this possibility, utilization of oleate $\left(\mathrm{C}_{18: 1}\right)$ and palmitate $\left(\mathrm{C}_{16: 0}\right)$ by $D$. amnigena (DSM 10 338) was tested in this study, but no growth was observed.

Microorganisms branching within the Syntrophobacteraceae group, corresponding to clones PS3 and PS6, have been previously detected in methanogenic palmitateenrichment cultures (Sousa et al., 2007b), and show high identity $(99 \%$ identity between clones PS3/PM82 and PS6/PM82) (Figs 2 and 3). Despite the high 16S rRNA 

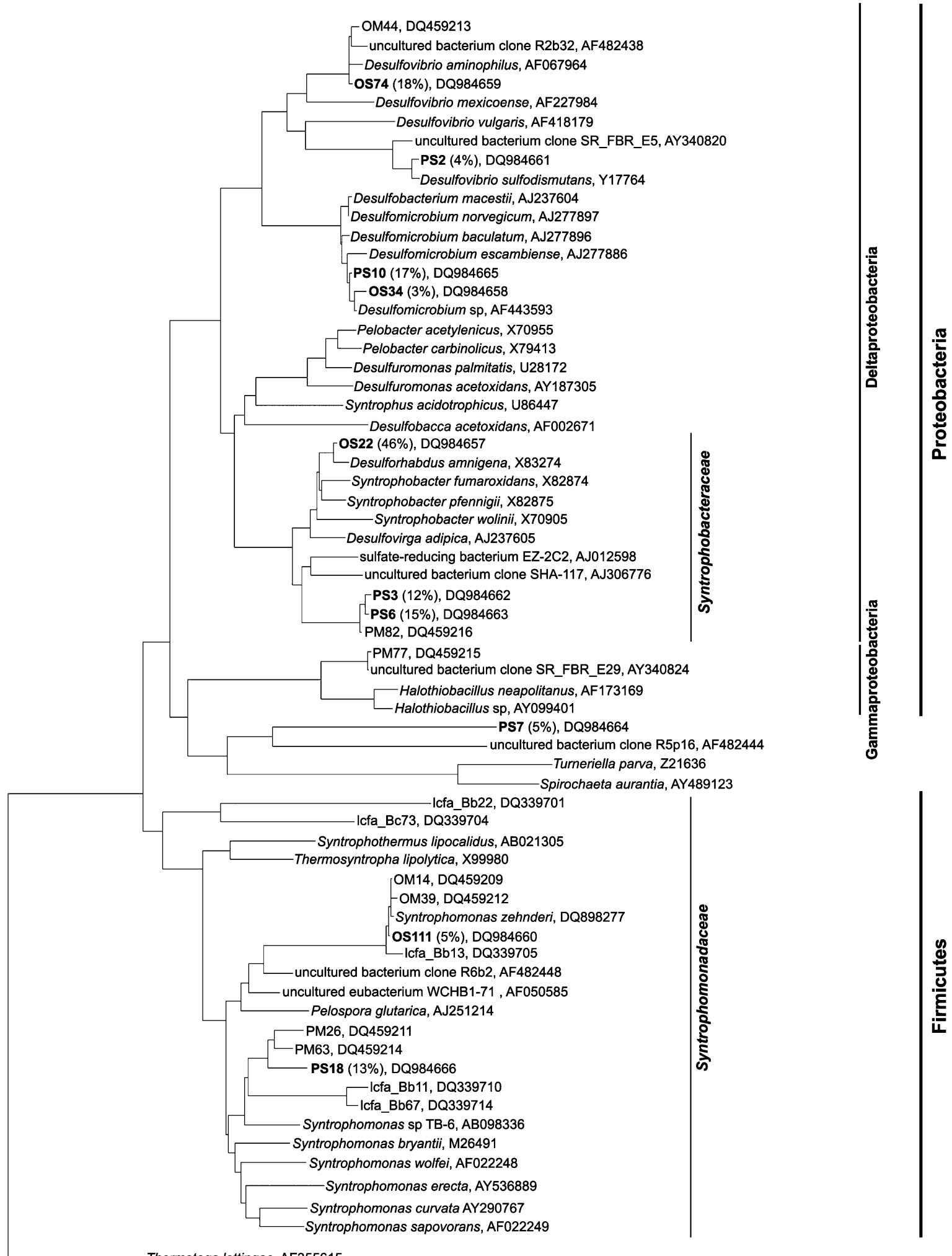

Thermotoga lettingae, AF355615 
Fig. 3. Phylogenetic tree of bacterial $16 \mathrm{~S}$ rRNA gene sequences retrieved from the stable oleate- and palmitate-enrichment cultures in the presence of sulfate (OS-7, PS-7). The tree was calculated using the ARB software package (Ludwig et al., 2004) and applying the neighbour-joining method (Saitou and Nei, 1987). Closely related sequences, with the respective GenBank accession number, are shown as reference. Thermotoga lettingae (AF355615) was used as out-group. Percentages shown in parenthesis correspond to the relative abundance of clones with identical ARDRA profiles (calculated from a total of 168 and 125 clones retrieved from OS-7 and PS-7 cultures respectively); all the other ARDRA profiles showed relative abundances of less than $3 \%$.

gene sequence similarity of clones PS3, PS6 and PS82, corresponding DGGE bands did not resolve at the same position (Fig. 2), indicating that these 16S rRNA gene sequences represent different bacterial strains. Identity of clones PS3 and PS6 with known SRB is rather low and closest related species are Syntrophobacter and Desulforhabdus (both with 90\% 16S rRNA gene identity).

\section{Principal component analysis of the bacterial DGGE profiles}

Comparison of the bacterial DGGE profiles from methanogenic and sulfate-reducing oleate- and palmitateenrichment series was performed by using principal component analysis (PCA, Fig. 4). It is important to note that the same inoculum sludge (sludge I) was used to start up methanogenic, OM and PM, and sulfate-reducing, OS and PS, enrichment series. Also, cultures OM-12 and $\mathrm{PM}-12$, used as inoculum for enrichments $\mathrm{OM} \rightarrow \mathrm{OS}$ and $\mathrm{PM} \rightarrow \mathrm{PS}$, were derived from enrichment series $\mathrm{OM}$ and $\mathrm{PM}$ respectively.
Results of the PCA of individual DGGE fingerprints further support the separation of profiles into two main clusters along the first principal component based on the substrate used as carbon and energy source (Fig. 4). In fact, the use of oleate $\left(\mathrm{C}_{18: 1}\right.$, unsaturated LCFA) or palmitate $\left(\mathrm{C}_{16: 0}\right.$, saturated LCFA), in four different enrichment series starting from the same inoculum, resulted in two clusters distantly related to the seed sludge, and rather associated to the substrate used. This suggests that the substrate supplied during the enrichment process played a more important role in the bacterial community differentiation than the absence/presence of sulfate.

\section{Relative abundance of archaea determined by real-time $P C R$}

Relative quantification of archaeal and bacterial 16S rRNA gene copy numbers in methanogenic and sulfatereducing enrichment cultures was achieved using domain-specific real-time PCR assays. It can be assumed that in anaerobic bioreactor sludges' most archaea are

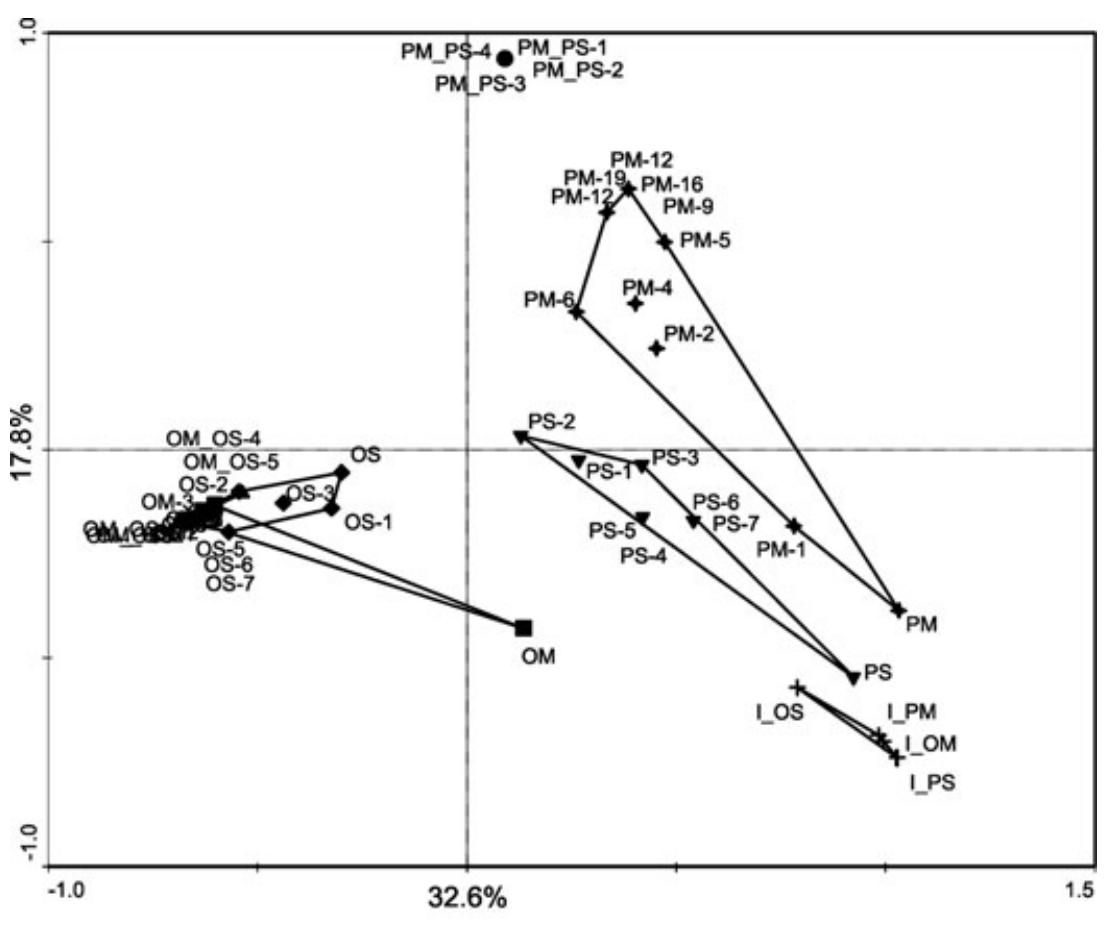

Fig. 4. Principal component analysis of DGGE profiles obtained after specific 165 rRNA gene amplification of genomic DNA from methanogenic and sulfate-reducing oleate- and palmitate-enrichment series (OM, PM, OS, PS, OM-OS and PM-PS enrichment series; numbers correspond to the number of transfers; all the enrichment series were started from the same inoculum sludge I). PC1 and PC2 explain $32.6 \%$ and $17.8 \%$ of the variation respectively. $\mathrm{PC} 1$ separates oleate from palmitate enrichments.

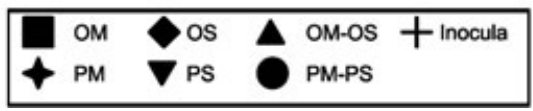

(C) 2008 The Authors

Journal compilation () 2008 Society for Applied Microbiology and Blackwell Publishing Ltd, Environmental Microbiology, 11, 68-80 
methanogens (Godon et al., 1997; Sekiguchi et al., 1998). Therefore relative archaeal copy numbers were used as indication for the presence or absence of methanogenic microorganisms in the different enrichment cultures as well as the inoculum used for the sulfate-reducing and methanogenic enrichment series.

A relative archaeal gene copy abundance of $41 \pm 5 \%$ was observed in the inoculum sludge. After enrichment of oleate and palmitate degraders, in the absence of sulfate, this number decreased to $23 \pm 11 \%$ and $15 \pm 4 \%$ respectively. The presence of sulfate in the medium completely inhibited the growth of archaea present in the initial inoculum sludge, and after seven successive transfers no archaea were detected in the sulfate-reducing oleateand palmitate-enrichment cultures. Moreover, when the methanogenic oleate- and palmitate-enrichment cultures were incubated and successively transferred in medium containing sulfate, archaeal 16S rRNA gene copy numbers sharply decreased to non-detectable values.

\section{Discussion}

This study describes the application of DGGE, cloning and sequencing to monitor the bacterial communities present in sulfate-reducing oleate- and palmitateenrichment cultures. Application of these molecular methods, in combination with cultivation, provided insights into bacterial populations involved in the anaerobic degradation of unsaturated (oleate, $\mathrm{C}_{18: 1}$ ) and saturated (palmitate, $\mathrm{C}_{16: 0}$ ) LCFA, in the presence of sulfate. Additionally, the use of real-time PCR showed the outcompetition of archaea present in methanogenic communities in the presence of sulfate as electron acceptor.

Considerable differences were found in the OS and PS cultures after enrichment, although both enrichment series were set up with the same inoculum (Fig. 1, lanes OS-7 and PS-7). Differentiation towards the LCFA used during the enrichment process was previously observed in methanogenic oleate and palmitate enrichments (Sousa et al., 2007b). In this study, PCA of the DGGE profiles from oleate- and palmitate-enrichment cultures confirmed this difference. In fact, application of PCA to DGGE profiles corresponding to oleate- and palmitateenrichment cultures, in which sulfate reduction (OS and PS) or methanogenesis (OM and PM) was favoured, showed that the organic substrate used during enrichment more strongly affected bacterial community structure than the presence or absence of sulfate itself (Fig. 4). It is interesting to note that oleate enrichments form a much more coherent and compact cluster compared with the different palmitate-enrichment series. One reason that might partially explain the differential clustering of the bacterial communities present in oleate- and palmitateenrichment cultures is the fact that the two LCFA used have different degree of chain saturation. Oleate $\left(\mathrm{C}_{18: 1}\right)$ is an unsaturated LCFA with a double bond at position C9, while palmitate $\left(C_{16: 0}\right)$ has a completely saturated chain. In fact, the ability of using unsaturated LCFA is not common to all fatty acid-oxidizing bacteria. To date, the only syntrophic acetogenic bacteria described as capable of utilizing mono- and/or polyunsaturated LCFA (with more than 12 carbon atoms) are Syntrophomonas sapovorans (Roy et al., 1986), Thermosyntrophica lipolytica (Svetlitshnyi et al., 1996), Syntrophomonas curvata (Zhang et al. 2004) and S. zehnderi (Sousa et al., 2007c). Degradation of unsaturated LCFA by fatty acid-oxidizing SRB was never reported.

Phylotypes affiliated with only a few genera were detected as predominant populations in the sulfatereducing oleate- and palmitate-enrichment cultures. These phylotypes included members of Syntrophomonas, as well as SRB closely affiliated with Desulfovibrio, Desulfomicrobium and Desulforhabdus (Fig. 3). Microorganisms branching within the Syntrophobacteraceae group, but with very low resemblance to yet described genera, were detected in the sulfate-reducing palmitateenrichment culture (clones PS3 and PS6, Fig. 3). According to the physiological characteristics of known Syntrophomonas spp., one can infer that these syntrophic fatty acid-oxidizing bacteria are responsible for oleate and palmitate $\beta$-oxidation. Hydrogen and acetate formed during this conversion could further be degraded via sulfate reduction by members of Desulfovibrio, Desulfomicrobium and Desulforhabdus genera, making the overall process thermodynamically feasible. Microorganisms corresponding to clones PS3 and PS6, detected in the sulfate-reducing palmitate-enrichment culture, have a less clear role in this system. Based on 16S rRNA gene analysis, distance of these microorganisms to characterized SRB is rather high; closest related microorganisms to clones PS3 and PS4 are Syntrophobacter and Desulforhabdus species, but with low $16 \mathrm{~S}$ rRNA identity (i.e. 90\%) (Fig. 3). A characteristic of almost all the described Syntrophobacter species is their ability to couple the oxidation of propionate to the reduction of sulfate (Wallrabenstein et al., 1994; 1995; Harmsen et al., 1998; Chen et al., 2005; Imachi et al., 2007), while D. amnigena (the only Desulforhabdus species described thus far) is a sulfate reducer that utilizes besides a number of other substrates also acetate for growth. Growth on LCFA by SRB belonging to these genera has never been reported, although $D$. amnigena is able to grow on short-chain fatty acids such as butyrate. Also it is not likely that bacteria corresponding to clones PS3 and PS6 are using propionate, because this is not an expected intermediary product from palmitate degradation. In this way, acetate is left as a probable substrate for bacteria corresponding to clones PS3 and PS6 although utilization of butyrate, a 
probable intermediate of palmitate degradation, is also a possible function of such bacteria. Uncertainty of the actual role of these bacteria during palmitate degradation warrants future efforts for their isolation and physiological characterization.

Sulfate-reducing bacteria known as fatty acids oxidizers were not detected in the oleate- and palmitate-enrichment cultures, in the presence of sulfate. Theoretically, SRB could compete with acetogenic syntrophic bacteria for common substrates. In the presence of butyrate and sulfate, some SRB such as Desulfobacterium autotrophicum (Brysch et al., 1987) and Desulfococcus multivorans (Stieb and Schink, 1989) have a kinetic growth advantage over the acetogenic Syntrophomonas wolfei (Mclnerney et al., 1979; 1981), implying that competition for butyrate may occur. Although these two SRB can also degrade LCFA, growth kinetic parameters in the presence of LCFA were never determined. In more complex environments little is known about this competition. Using most probable number counting, Visser and colleagues (1993) found that syntrophic butyrate oxidizers were able to compete effectively with sulfate reducers for butyrate, even at an excess of sulfate. No competition studies between acetogens and SRB were ever performed for degradation of fatty acids with more than four carbon atoms.

Overall, characterization of the bacterial communities present in oleate and palmitate enrichments in the presence (this study) and absence (Sousa et al., 2007b) of sulfate reinforced the importance of syntrophic fatty acidoxidizing bacteria during anaerobic LCFA degradation. Previous studies on LCFA degradation in methanogenic bioreactors have identified members of this group as important players in the process (Pereira et al., 2002b; Sousa et al., 2007a). However, this is the first report that shows a predominance of syntrophic fatty acid-oxidizing bacteria in LCFA-degrading cultures in the presence of sulfate. The major difference found between methanogenic and sulfate-reducing LCFA enrichments is therefore not related to the predominant groups of LCFA-degrading bacteria, but to the hydrogen- and acetate-consuming microorganisms present. In the presence of sulfate, hydrogen- and acetate-consuming methanogens are outcompeted by sulfate reducers, as was verified after the addition of sulfate to methanogenic oleate- and palmitateenrichment cultures. After incubation (and successive subculturing) in media with sulfate of methanogenic oleate- and palmitate-enrichment cultures, predominant bands corresponding to Desulfovibrio- and Desulfohabdus-related microorganisms appeared in the DGGE profiles of these samples (Figs 2 and 3). Moreover, no archaea could be detected by real-time PCR in samples after incubation with sulfate, confirming that the methanogenic community was out-competed. Thermodynamically, conversion of hydrogen and acetate via sulfate reduction is more favourable than their conversion via methanogenesis, as follows $\left[\Delta G^{0 \prime}\right.$ values were taken from Thauer and colleagues (1977)]:

Methanogenic reactions:

$$
\begin{gathered}
4 \mathrm{H}_{2}+\mathrm{HCO}_{3}^{-}+\mathrm{H}^{+} \rightarrow \mathrm{CH}_{4}+3 \mathrm{H}_{2} \mathrm{O} \quad \Delta \mathrm{G}^{0 \prime}=-136 \mathrm{~kJ} \mathrm{reaction}^{-1} \\
\text { Acetate }^{-}+\mathrm{H}_{2} \mathrm{O} \rightarrow \mathrm{CH}_{4}+\mathrm{HCO}_{3}^{-} \quad \Delta \mathrm{G}^{0 \prime}=-31 \mathrm{~kJ} \text { reaction }
\end{gathered}
$$

Sulfate reduction reactions:

$$
\begin{gathered}
4 \mathrm{H}_{2}+\mathrm{SO}_{4}{ }^{2-}+\mathrm{H}^{+} \rightarrow \mathrm{HS}^{-}+4 \mathrm{H}_{2} \mathrm{O} \quad \Delta \mathrm{G}^{0 \prime}=-152 \mathrm{~kJ} \text { reaction } \\
\end{gathered}
$$

According to the $\Delta G^{0 \prime}$ values, SRB should out-compete methanogens for both hydrogen and acetate utilization. Additionally, kinetic properties of SRB and methanogens also indicate that the outcome of the competition for these common substrates might be in favour of SRB (Stams et al., 2005). Sulfate-reducing bacteria have a higher affinity for hydrogen than methanogens, which is related to the fact that sulfate reducers can keep the hydrogen partial pressure lower than methanogens (Mclnerney et al., 1981). This higher affinity for hydrogen coupled with a higher biomass yield indicates that SRB can effectively out-compete methanogens, when growing at limiting substrate levels (Colleran et al., 1995). This is consistent with the fact that in oleate- and palmitate-enrichment cultures cultivated with sulfate hydrogenotrophic SRB, namely Desulfovibrio- and Desulfomicrobium-related species, took over methanogens during hydrogen scavenging. Concerning acetate utilization, postulates about kinetic properties from SRB and methanogens are not so straightforward. Actually, kinetic properties of Methanosaeta species do not differ significantly from the ones shown by acetate-consuming SRB, namely $D$. amnigena (Oude Elferink et al., 1998). A closely related microorganism to $D$. amnigena is predominantly present in sulfate-reducing oleate-enrichment cultures. However, its advantage might be related with a higher tolerance to oleate when compared with methanogens. Note that in methanogenic oleate-enrichment cultures acetate was accumulating in the medium meaning that aceticlastic methanogens were virtually not present or inactive (Sousa et al., 2007b); also, aceticlastic methanogens are reported as being more affected by the presence of LCFA (Hanaki et al., 1981; Koster and Cramer, 1987; Rinzema et al., 1994; Lalman and Bagley, 2001). In the sulfate-reducing palmitateenrichment cultures no evident acetate-degrading SRB could be identified. However, as discussed above, acetate is most likely used by microorganisms corresponding to clones PS3 and PS6. However, the phylogenetic distance of these microorganisms to known SRB does not permit a clear view on their physiologic and kinetic properties. The 
observed rapid onset of sulfate reduction in the enrichment cultures cultivated with sulfate could be related to the presence of non-sulfate-dependent SRB in the methanogenic enrichment cultures (e.g. clone OM44, Figs 2 and 3). Although these SRB do not depend on sulfate reduction, its use as electron acceptor might be metabolically more favourable.

In conclusion, enrichment of anaerobic sludge on oleate $\left(\mathrm{C}_{18: 1}\right.$, unsaturated LCFA) and palmitate $\left(\mathrm{C}_{16: 0}\right.$, saturated LCFA) in the presence of sulfate resulted in the development of two distinct syntrophic communities. Two major groups - syntrophic LCFA-degrading bacteria and sulfate-reducing hydrogen and acetate consumers could be identified in the enrichment cultures. No known sulfate-reducing LCFA-degrading bacteria were detected in the oleate- and palmitate-enrichment cultures. Sulfatereducing hydrogen and acetate consumers were shown to rapidly out-compete methanogens when sulfate is present. Further study of the microbial populations in different anaerobic reactors treating lipids/LCFA-based wastewaters, in the presence of sulfate, might be important to model competition between methanogens and SRB in this type of systems.

\section{Experimental procedures}

\section{Medium composition and cultivation}

A bicarbonate-buffered mineral salt medium (Stams et al., 1993) was used for enrichment. Serum bottles were sealed with butyl rubber septa and aluminium crimp caps, and the headspace was flushed with a mixture of $\mathrm{N}_{2} / \mathrm{CO}_{2}$ (80:20; $\left.1.7 \times 10^{5} \mathrm{~Pa}\right)$. Mineral medium was reduced with $0.8 \mathrm{mM}$ sodium sulfide $\left(\mathrm{Na}_{2} \mathrm{~S} \cdot 9 \mathrm{H}_{2} \mathrm{O}\right)$ before inoculation. Substrates supplied as sodium salts and sodium sulfate were added from sterile stock solutions to the required final concentrations. All inoculations and transfers were performed aseptically using sterile syringes and needles, and all cultures were incubated statically at $37^{\circ} \mathrm{C}$ in the dark.

\section{Sulfate-reducing oleate- and palmitate-enrichment cultures}

Anaerobic sludge, acclimated to LCFA, was used for the start-up of sulfate-reducing oleate- and palmitate-enrichment cultures. Sludge acclimation to LCFA consisted of a continuous-batch process. First, anaerobic sludge (obtained from a municipal sludge digester) was continuously loaded with a synthetic skim milk/oleate-based effluent. After 77 days of continuous loading, sludge was collected from the reactor, washed twice with anaerobic basal medium to remove any dissolved substrates that were present in the medium and incubated in batch vials. During batch incubation no carbon source was supplemented to the sludge, so that biomass-associated LCFA could be completely degraded. After approximately 10 days of batch incubation, biomass was washed and concentrated with anaerobic medium and used to inoculate enrichment series OS and PS (concurrently with enrichment series OM and PM; Sousa et al., 2007b). During the acclimation process (that lasted for 87 days) no sulfate or other external electron acceptor was supplemented to the sludge so pressure directed the system towards methanogenesis. Enrichment series $\mathrm{OM} \rightarrow \mathrm{OS}$ and $\mathrm{PM} \rightarrow \mathrm{PS}$ were started by transferring highly enriched methanogenic oleate- and palmitate-degrading cultures (cultures OM-12 and $\mathrm{PM}-12$ deriving from enrichment series $\mathrm{OM}$ and $\mathrm{PM}$; Sousa et al., 2007b) to medium containing oleate and palmitate, respectively, plus sulfate. Sulfate-reducing enrichment series were further developed by successive transfers of active cultures (10\% v/v) into new medium containing $10 \mathrm{mM}$ sodium sulfate plus $1 \mathrm{mM}$ oleate $\left(\mathrm{C}_{18: 1}\right.$, unsaturated LCFA) or $1 \mathrm{mM}$ palmitate $\left(\mathrm{C}_{16: 0}\right.$, saturated LCFA) (see Fig. $\mathrm{S} 1$ for a schematic representation of the experiment set-up). Oleate and palmitate (sodium salts of fatty acids) (Fluka, Riedel-deHaën, Sigma-Aldrich, Seelze, Germany) were added to the medium from sterile stock solutions to a final concentration of $1 \mathrm{mM}$, and served as the sole source of carbon and energy. LCFA degradation was easily observed by the clearing of LCFA in solution and/or suspension (addition of oleate or palmitate to the medium causes an immediate turbid-white appearance). Sulfide production and methane formation during LCFA degradation were detected using a colour indication method and gas chromatography, respectively, as described below.

\section{Oleate and palmitate utilization by a SRB}

Growth of D. amnigena DSM 10338 on oleate or palmitate was tested using the same basal medium and conditions applied to the enrichment cultures ( $1 \mathrm{mM}$ oleate/palmitate plus $10 \mathrm{mM}$ sulfate, $37^{\circ} \mathrm{C}$ ). Desulforhabdus amnigena DSM 10338 was obtained from the Deutsche Sammlung von Mikroorganismen und Zellkulturen (DSMZ), Braunschweig, Germany.

\section{Analytical methods}

Before each transfer of the enrichment cultures, sulfide was detected using a colour indicator method with copper sulfate. Briefly, $0.1 \mathrm{ml}$ of liquid sample was added to $1 \mathrm{ml}$ of an acidic copper sulfate solution containing $1.3 \mathrm{~g}$ of $\mathrm{CuSO}_{4} \cdot 5 \mathrm{H}_{2} \mathrm{O}$ and $4.2 \mathrm{ml}$ of concentrated $\mathrm{HCl}(37 \%)$ per litre. Colour development from colourless/yellow to light/dark brown, due to the formation of CuS colloids, is indicative of sulfide presence at concentrations ranging from 1 to $10 \mathrm{mM}$. Stable sulfatereducing oleate- and palmitate-enrichment cultures were quantitatively analysed for sulfide and VFA. Sulfide was determined colorimetrically after reaction with $N, N$-dimethyl$p$-phenylenediamine oxalate according the method described by Trüper and Schlegel (1964). Volatile fatty acids were analysed by high-pressure liquid chromatography from centrifuged $(10000 \mathrm{~g}, 10 \mathrm{~min})$ samples of the culture media. Volatile fatty acids were measured with a Polyspher OA HY column (300 to $6.5 \mathrm{~mm}$; Merck, Darmstadt, Germany) and an RI SE-61 refractive index detector (Shodex, Tokyo, Japan). The mobile phase was $0.01 \mathrm{~N} \mathrm{H}_{2} \mathrm{SO}_{4}$ at a flow rate of $0.6 \mathrm{ml} \mathrm{min}^{-1}$. The column temperature was $60^{\circ} \mathrm{C}$. Gas in the bottles' headspace was checked for the presence of methane. Gas samples were analysed by gas chromatogra- 
phy with a GC-14B apparatus (Shimadzu, Kyoto, Japan) equipped with a packed column (molsieve 13X 60/80 mesh, $2 \mathrm{~m}$ length, $2.4 \mathrm{~mm}$ internal diameter; Varian, Middelburg, the Netherlands) and a TCD detector. The oven temperature was $100^{\circ} \mathrm{C}$ and the injector and detector temperatures were $90^{\circ} \mathrm{C}$ and $150^{\circ} \mathrm{C}$ respectively. Argon was the carrier gas at a flow rate of $30 \mathrm{ml} \mathrm{min}^{-1}$.

\section{DNA extraction and PCR amplification}

Approximately $10 \mathrm{ml}$ aliquots of well-homogenized microbial cultures were immediately concentrated by centrifugation (10 $509 \mathrm{~g}, 5 \mathrm{~min}$ ) at the time of sampling, frozen and stored at $-20^{\circ} \mathrm{C}$. Total genomic DNA was extracted using a FastDNA SPIN Kit for Soil (Qbiogene, Carlsbad, CA, USA).

The 16S rRNA genes were amplified by PCR using a Taq DNA Polymerase kit (Life Technologies, Gaithersburg, MD, USA) with primers targeting conserved domains. Reaction preparation and thermocycling programmes used for amplification were as those described previously (Sousa et al., 2007a). All primers used were synthesized commercially by MWG-Biotech (Ebersberg, Germany) (Table S1). Bacterial 16S rRNA genes were selectively amplified for cloning using primers Bact27-f and Uni1492-r. For DGGE analysis PCR products were generated using primers U968GC-f and L1401-r, targeting the V6 to V8 region of bacterial 16S rRNA. Size and yield of PCR products were estimated using a 100 bp DNA ladder (MBI Fermentas, Vilnius, Lithuania) by $1 \%$ agarose gel $(\mathrm{w} / \mathrm{v})$ electrophoresis and ethidium bromide staining.

\section{DGGE analysis}

DGGE analysis of the amplicons was performed as described by Zoetendal and colleagues (2001) by using the Dcode system (Bio-Rad, Hercules, CA, USA) with $8 \%$ (v/v) polyacrylamide gels and a denaturant gradient of $30-60 \%$. A $100 \%$ denaturing solution was defined as $7 \mathrm{M}$ urea and $40 \%$ formamide. Electrophoresis was performed for $16 \mathrm{~h}$ at $85 \mathrm{~V}$ in a $0.5 \times \mathrm{TAE}$ buffer at $60^{\circ} \mathrm{C}$. DGGE gels were stained with $\mathrm{AgNO}_{3}$ as described by Sanguinetti and colleagues (1994), scanned at $400 \mathrm{dpi}$, and the DGGE profiles were compared using the GelCompar $\mathrm{II}^{\mathrm{TM}}$ software (Applied Maths BVBA, Sint-Martens-Latem, Belgium). After image normalization, bands were defined for each sample using the bands search algorithm within the programme. Similarity indices of the compared profiles were calculated from the densitometric curves of the scanned DGGE profiles by using the Pearson product-moment correlation coefficient (Hane et al., 1993). Principal component analysis of the DGGE profiles was performed as implemented in the CANOCO 4.5 software package (Biometris, Wageningen, the Netherlands), using binary band matching values. Peak heights in the densitometric curves were also used to determine the diversity indices based on the Shannon-Wiener diversity index, calculated as follows:

$$
H=-\sum\left[P_{i} \ln \left(P_{i}\right)\right]
$$

where $H$ is the diversity index and $P_{i}$ is the importance probability of the bands in a lane $\left(P_{i}=n_{i} / n\right.$, where $n_{i}$ is the height of an individual peak and $n$ is the sum of all peak heights in the densitometric curves).

\section{Cloning and sequencing of PCR-amplified products}

Polymerase chain reaction amplicons were purified with the QIAquick PCR purification kit (Qiagen $\mathrm{GmbH}$, Hilden, Germany) according to the manufacturer's instructions and cloned into Escherichia coli JM109 (Invitrogen, Breda, the Netherlands) by using the Promega pGEM-T Easy vector system (Promega, Madison, WI, USA). After PCR amplification with the pGEM-T-specific primers PG1-f and PG2-r (Table S1), amplicons of the correct size were screened by ARDRA. Restriction enzymes Mspl, Cfol and Alul (Promega, Madison, WI, USA) were used for this analysis, in the presence of $0.1 \mathrm{mg} \mathrm{ml}^{-1}$ acetylated bovine serum albumin (BSA). The restriction fragments were analysed by electrophoresis in a $4 \%$ agarose gel $(\mathrm{w} / \mathrm{v})$ and visualized with ethidium bromide. Plasmids of selected transformants, with different ARDRA pattern and corresponding to prominent bands in the DGGE community fingerprint, were purified using the QIAquick PCR purification kit (Qiagen, Hilden, Germany) and subjected to DNA sequence analysis. Sequencing reactions were performed at BaseClear (Leiden, the Netherlands) using pGEM-T vector-targeted sequencing primers, SP6 and T7, and 16S rRNA gene-targeted internal primer, Uni-533-f and Bact-1100-r (Table S1). Sequences were assembled and aligned using the programs EditSeq and SeqMan II (DNAstar, Madison, WI, USA). Consensus sequences were checked for potential chimera artefacts by the CHECK_CHIMERA program of the Ribosomal Database Project II (RDP release 8.1, http:// rdp8.cme.msu.edu/html/) (Cole et al., 2003).

\section{Phylogenetic analysis of $16 S$ rRNA sequences}

Similarity searches for the 16S rRNA gene sequences of enrichment culture-derived clones were performed using the NCBI BLAST search program within the GenBank database (Altschul et al., 1990). Alignment of the $16 \mathrm{~S}$ rRNA sequences was performed by using the FastAligner V1.03 tool of the ARB program package (Ludwig et al., 2004). The resulting alignments were manually checked and corrected when necessary, and unambiguously aligned nucleotide positions were used for construction of a 16S rRNA gene-based phylogenetic tree, using the neighbour-joining method (Saitou and $\mathrm{Nei}, 1987$ ). Phylogenetic placement was performed in comparison with reference sequences with Felsenstein correction and application of appropriate filters at the respective phylum level.

\section{Real-time $P C R$}

Real-time PCR quantification of archaeal and bacterial $16 S$ rRNA gene copies in sludge samples was carried using the iQ5 real-time PCR detection system and the iQ SYBR Green Supermix Kit (Bio-Rad, Hercules, CA, USA). Archaeal and bacterial 16S rRNA genes were amplified with primer sets ARC787-f/ARC1059-r and BAC338-f/BAC805-r (Table S1), following the procedure previously described by Sousa and colleagues (2007a). Relative abundance of archaea (\%) in 
the analysed samples was calculated using the following formula: Archaea copies/(Archaea copies + Bacteria copies) $\times 100$.

\section{Nucleotide sequence accession numbers}

Nearly complete $16 S$ rRNA sequences of the 16S rRNA gene clones have been deposited in the GenBank database under Accession No. DQ984657 to DQ984666.

\section{Acknowledgements}

This work was possible through grants attributed to D.Z. Sousa by Fundação para a Ciência e Tecnologia (FCT) and Fundo Social Europeu (FSE) (SFRH/BD/8726/2002), and by the Wageningen Institute for Environmental and Climate Research (WIMEK).

\section{References}

Alphenaar, P.A., Visser, A., and Lettinga, G. (1993) The effect of liquid upward velocity and hydraulic retention time on granulation in UASB reactors treating wastewater with a high sulfate content. Bioresour Technol 43: 249-258.

Altschul, S.F., Gish, W., Miller, W., Meyers, E.W., and Lipman, D.J. (1990) Basic local alignment search tool. $J$ Mol Biol 215: 403-410.

Beeder, J., Torsvik, T., and Lien, T.L. (1995) Thermodesulforhabdus norvegicus gen. nov., sp. nov., a novel thermophilic sulfate-reducing bacterium from oil field water. Arch Microbiol 164: 331-336.

Brysch, K., Schneider, C., Fuchs, G., and Widdel, F. (1987) Lithoautotrophic growth of sulfate-reducing bacteria, and description of Desulfobacterium autotrophicum gen. nov., sp. nov. Arch Microbiol 148: 264-274.

Chen, S.Y., Liu, X.L., and Dong, X.Z. (2005) Syntrophobacter sulfatireducens sp. nov., a novel syntrophic, propionateoxidizing bacterium isolated from UASB reactors. Int J Syst Evol Microbiol 55: 1319-1324.

Cole, J.R., Chai, B., Marsh, T.L., Farris, R.J., Wang, Q., Kulam, S.A., et al. (2003) The Ribosomal Database Project (RDP-II): previewing a new autoaligner that allows regular updates and the new prokaryotic taxonomy. Nucleic Acids Res 31: 442-443.

Colleran, E., Finnegan, S., and Lens, P. (1995) Anaerobic treatment of sulfate-containing waste streams. Antonie Van Leeuwenhoek 67: 29-46.

Godon, J.J., Zumstein, E., Dabert, P., Habouzit, F., and Moletta, R. (1997) Molecular microbial diversity of an anaerobic digestor as determined by small-subunit rDNA sequence analysis. Appl Environ Microbiol 63: 2802-2813.

Gupta, A., Flora, J.R.V., Gupta, M., Sayles, G.D., and Suidan, M.T. (1994) Methanogenesis and sulfate reduction in chemostats. 1. Kinetic studies and experiments. Water Res 28: 781-793.

Hanaki, K., Nagase, M., and Matsuo, T. (1981) Mechanism of inhibition caused by long-chain fatty acids in anaerobic digestion process. Biotechnol Bioeng 23: 1591-1610.

Hane, B.G., Jager, K., and Drexler, H.G. (1993) The Pearson product-moment correlation coefficient is better suited for identification of DNA fingerprint profiles than band matching algorithms. Electrophoresis 14: 967-972.

Harada, H., Uemura, S., and Momonoi, K. (1994) Interaction between sulfate-reducing bacteria and methane-producing bacteria in UASB reactors fed with low strength wastes containing different levels of sulfate. Water Res 28: 355367.

Harmsen, H.J.M., Van Kuijk, B.L.M., Plugge, C.M., Akkermans, A.D.L., de Vos, W.M., and Stams, A.J.M. (1998) Syntrophobacter fumaroxidans sp. nov., a syntrophic propionate-degrading sulfate-reducing bacterium. Int $J$ Syst Bacteriol 48: 1383-1387.

Imachi, H., Sakai, S., Ohashi, A., Harada, H., Hanada, S., Kamagata, Y., and Sekiguchi, Y. (2007) Pelotomaculum propionicicum sp. nov., an anaerobic, mesophilic, obligately syntrophic propionate-oxidizing bacterium. Int J Syst Evol Microbiol 57: 1487-1492.

Isa, Z., Grusenmeyer, S., and Verstraete, W. (1986) Sulfate reduction relative to methane production in high-rate anaerobic digestion: microbiological aspects. Appl Environ Microbiol 51: 580-587.

Koster, I.W., and Cramer, A. (1987) Inhibition of methanogenesis from acetate in granular sludge by long-chain fatty acids. Appl Environ Microbiol 53: 403-409.

Lalman, J.A., and Bagley, D.M. (2001) Anaerobic degradation and methanogenic inhibitory effects of oleic and stearic acids. Water Res 35: 2975-2983.

Ludwig, W., Strunk, O., Westram, R., Richter, L., Meier, H., Yadhukumar, et al. (2004) ARB: a software environment for sequence data. Nucleic Acids Res 32: 1363-1371.

Mclnerney, M.J., Bryant, M.P., and Pfennig, N. (1979) Anaerobic bacterium that degrades fatty-acids in syntrophic association with methanogens. Arch Microbiol 122: 129-135.

Mclnerney, M.J., Bryant, M.P., Hespell, R.B., and Costerton, J.W. (1981) Syntrophomonas wolfei gen. nov. sp. nov., an anaerobic, syntrophic, fatty-acid oxidizing bacterium. Appl Environ Microbiol 41: 1029-1039.

O'Flaherty, V., Lens, P., Leahy, B., and Colleran, E. (1998) Long-term competition between sulphate-reducing and methane-producing bacteria during full-scale anaerobic treatment of citric acid production wastewater. Water Res 32: 815-825.

Omil, F., Lens, P., Hulshoff Pol, L.W., and Lettinga, G. (1996) Effect of upward velocity and sulphide concentration on volatile fatty acid degradation in a sulphidogenic granular sludge reactor. Process Biochem 31: 699-710.

Oude Elferink, S.J.W.H., Maas, R.N., Harmsen, H.J.M., and Stams, A.J.M. (1995) Desulforhabdus amnigenus gen. nov., sp. nov., a sulfate reducer isolated from anaerobic granular sludge. Arch Microbiol 164: 119-124.

Oude Elferink, S.J.W.H., Luppens, S.B.I., Marcelis, C.L.M., and Stams, A.J.M. (1998) Kinetics of acetate oxidation by two sulfate reducers isolated from anaerobic granular sludge. Appl Environ Microbiol 64: 2301-2303.

Pereira, M.A., Pires, O.C., Mota, M., and Alves, M.M. (2002a) Anaerobic degradation of oleic acid by suspended and granular sludge: identification of palmitic acid as a key intermediate. Water Sci Technol 45: 139-144.

Pereira, M.A., Roest, K., Stams, A.J.M., Mota, M., Alves, M., and Akkermans, A.D.L. (2002b) Molecular monitoring of 
microbial diversity in expanded granular sludge bed (EGSB) reactors treating oleic acid. FEMS Microbiol Ecol 41: 95-103.

Pereira, M.A., Cavaleiro, A.J., Mota, M., and Alves, M.M. (2003) Accumulation of long chain fatty acids onto anaerobic sludge under steady state and shock loading conditions: effect on acetogenic and methanogenic activity. Water Sci Technol 48: 33-40.

Pereira, M.A., Sousa, D.Z., Mota, M., and Alves, M.M. (2004) Mineralization of LCFA associated with anaerobic sludge: kinetics, enhancement of methanogenic activity, and effect of VFA. Biotechnol Bioeng 88: 502-511.

Rabus, R., Hansen, T., and Widdel, F. (2006) Dissimilatory sulfate- and sulfur-reducing prokaryotes. In The Prokaryotes. Dworkin, M., Falkow, S., Rosenberg, E., Schleifer, K.-H., and Stackebrandt, E. (eds). New York, USA: Springer, pp. 659-768.

Rees, G.N., and Patel, B.K.C. (2001) Desulforegula conservatrix gen. nov., sp nov., a long-chain fatty acid-oxidizing, sulfate-reducing bacterium isolated from sediments of a freshwater lake. Int J Syst Evol Microbiol 51: 1911-1916.

Rinzema, A., Boone, M., van Knippenberg, K., and Lettinga, G. (1994) Bactericidal effect of long chain fatty acids in anaerobic digestion. Water Environ Res 66: 40-49.

Roy, F., Samain, E., Dubourguier, H.C., and Albagnac, G. (1986) Synthrophomonas sapovorans sp. nov., a new obligately proton reducing anaerobe oxidizing saturated and unsaturated long-chain fatty acids. Arch Microbiol 145: 142-147.

Saitou, N., and Nei, M. (1987) The neighbor-joining method: a new method for reconstructing phylogenetic trees. Mol Biol Evol 4: 406-425.

Sanguinetti, C.J., Dias Neto, E., and Simpson, A.J. (1994) Rapid silver staining and recovery of PCR products separated on polyacrylamide gels. Biotechniques 17: 914-921.

Sekiguchi, Y., Kamagata, Y., Syutsubo, K., Ohashi, A., Harada, H., and Nakamura, K. (1998) Phylogenetic diversity of mesophilic and thermophilic granular sludges determined by $16 \mathrm{~S}$ rRNA gene analysis. Microbiology 144: 2655-2665.

Sousa, D.Z., Pereira, M.A., Smidt, H., Stams, A.J.M., and Alves, M.M. (2007a) Molecular assessment of complex microbial communities degrading long chain fatty acids in methanogenic bioreactors. FEMS Microbiol Ecol 60: 252265.

Sousa, D.Z., Pereira, M.A., Stams, A.J.M., Alves, M.M., and Smidt, H. (2007b) Microbial communities involved in anaerobic degradation of unsaturated or saturated long chain fatty acids (LCFA). Appl Environ Microbiol 73: 10541064.

Sousa, D.Z., Smidt, H., Alves, M.M., and Stams, A.J.M. (2007c) Syntrophomonas zehnderi sp. nov., an anaerobe that degrades long chain fatty acids in co-culture with Methanobacterium formicicum. Int J Syst Evol Microbiol 57: 609-615.

Stams, A.J.M., Vandijk, J.B., Dijkema, C., and Plugge, C.M. (1993) Growth of syntrophic propionate-oxidizing bacteria with fumarate in the absence of methanogenic bacteria. Appl Environ Microbiol 59: 1114-1119.

Stams, A.J.M., Plugge, C.M., De Bok, F.A.M., van Houten, B.H.G.W., Lens, P., Dijkman, H., and Weijma, J. (2005)
Metabolic interactions in methanogenic and sulfatereducing bioreactors. Water Sci Technol 52: 13-20.

Stieb, M., and Schink, B. (1989) Anaerobic degradation of isobutyrate by methanogenic enrichment cultures and by a Desulfococcus multivorans strain. Arch Microbiol 151: 126-132.

Stucki, G., Hanselmann, K.W., and Hurzeler, R.A. (1993) Biological sulfuric acid transformation: reactor design and process optimization. Biotechnol Bioeng 41: 303-315.

Svetlitshnyi, V., Rainey, F., and Wiegel, J. (1996) Thermosyntropha lipolytica gen. nov., sp. nov., a lipolytic, anaerobic, alkalitolerant, thermophilic bacterium utilizing short- and long-chain fatty acids in syntrophic coculture with a methanogenic archaeum. Int $J$ Syst Bacteriol 46: 1131-1137.

Thauer, R.K., Jungermann, K., and Decker, K. (1977) Energy conservation in chemotropic anaerobic bacteria. Bacteriol Rev 41: 100-180.

Trüper, H.G., and Schlegel, H.G. (1964) Sulphur metabolism in Thiorhodaceae. 1. Quantitative measurements on growing cells of Chromatium okenii. Antonie Van Leeuwenhoek 30: 225-238.

Visser, A., Beeksma, I., van der Zee, F., Stams, A.J.M., and Lettinga, G. (1993) Anaerobic degradation of volatile fattyacids at different sulfate concentrations. Appl Microbiol Biotechnol 40: 549-556.

Wallrabenstein, C., Hauschild, E., and Schink, B. (1994) Pure culture and cytological properties of Syntrophobacter wolinii. FEMS Microbiol Lett 123: 249-254.

Wallrabenstein, C., Hauschild, E., and Schink, B. (1995) Syntrophobacter pfennigii sp. nov, new syntrophically propionate-oxidizing anaerobe growing in pure culture with propionate and sulfate. Arch Microbiol 164: 346-352.

Zhang, C.Y., Liu, X.L., and Dong, X.Z. (2004) Syntrophomonas curvata sp. nov., an anaerobe that degrades fatty acids in co-culture with methanogens. Int $J$ Syst Evol Microbiol 54: 969-973.

Zoetendal, E.G., Akkermans, A.D.L., Akkermans van Vliet, W.M., de Visser, J.A.G.M., and de Vos, W.M. (2001) The host genotype affects the bacterial community in the human gastrointestinal tract. Microb Ecol Health Dis 13: $129-134$.

\section{Supporting information}

Additional Supporting Information may be found in the online version of this article:

Fig. S1. Flowsheet diagram of the experimental procedure used for obtaining the four distinct enrichment series presented in this study: two oleate-enrichment series - OS and $\mathrm{OM} \rightarrow \mathrm{OS}$, and two palmitate-enrichment series - PS and $\mathrm{PM} \rightarrow \mathrm{PS}$. OS and PS enrichment series derive directly from sludge I [previously adapted to LCFA as described by Sousa and colleagues (2007b)]. This sludge was also used to start up enrichment series OM and PM, under methanogenic conditions (Sousa et al., 2007b). Stable methanogenic enrichment cultures, OM-12 and PM-12, were further exposed to contact with sulfate in enrichment series $\mathrm{OM} \rightarrow \mathrm{OS}$ and $\mathrm{PM} \rightarrow \mathrm{PS}$.

Fig. S2. Palmitate- and oleate-enrichment cultures before (PS-7/OS-7) and after (PS-6/OS-6) incubation. White-turbid 
80 D. Z. Sousa et al.

aspect of the liquid medium in bottles PS-7 and OS-7 is due to the palmitate/oleate in suspension; after 3-4 weeks of incubation liquid medium becomes transparent, as can be observed in bottles PS- 6 and OS-6, indicative of microbial degradation of the LCFA.

Fig. S3. Pathways suggested in the present work for oleate and palmitate degradation under (A) methanogenic and $(B)$ sulfate-reducing conditions. In a first step, analogous in methanogenic and sulfate-reducing enrichments, oleate and palmitate are converted to acetate and hydrogen by acetogenic syntrophic bacteria. A second step, in which hydrogen and acetate are consumed, makes LCFA oxidation thermodynamically feasible. In the absence of sulfate hydrogen and acetate are utilized by methanogenic archaea while in the presence of sulfate this is performed by sulfate-reducing bacteria.

Table S1. Oligonucleotides used in this study.

Please note: Blackwell Publishing are not responsible for the content or functionality of any supporting materials supplied by the authors. Any queries (other than missing material) should be directed to the corresponding author for the article. 